\title{
Intelligent Traffic Light System to Prioritized Emergency Purpose Vehicles based on Wireless Sensor Network
}

\author{
Amnesh Goel \\ Amity School Of Engineering \\ \& Technology, Amity University, \\ Noida (U.P.), India
}

\author{
Sukanya Ray \\ Amity School Of Engineering \\ \& Technology, Amity University, \\ Noida (U.P.), India
}

\author{
Nidhi Chandra \\ Amity School Of Engineering \\ \& Technology, Amity University, \\ Noida (U.P.), India
}

\begin{abstract}
The use of Wireless Sensor Network (WSN) has proved to be a very beneficial in the design of adaptive and dynamic traffic light intersection system that will minimize the waiting time of vehicles and also manage the traffic load at the intersection adaptively. In this paper, we propose an adaptive traffic intersection system based on Wireless Sensor Network where the traffic light of one intersection can communicate with the traffic light of the next neighboring intersections and traffic clearance will be prioritized for special vehicles with the help of sensors.
\end{abstract}

General Terms: Wireless Sensor Network.

Keywords: Adaptive traffic light, ITS, Dynamic.

\section{INTRODUCTION}

Nowadays Wireless Sensor Networks (WSN) has been applied in various domains like weather monitoring, military, home automation, health care monitoring, security and safety etc. or in a nut shell one can say wireless sensor network can be applied in most of the domains. Traffic Signal System or traffic monitoring is a vast domain where WSN can be applied to gather information about the incoming flow of traffic, traffic load on a particular road, traffic load at particular period of time (peak hours) and in vehicle prioritization. WSN installed along a road can be utilized to control the traffic load on roads and at traffic intersections.

The sensor nodes that are to be deployed along the road are small in size and have low energy consumption.[1] These sensors run on both battery power as well as solar energy. They have the capability to draw solar energy so that they can use sunlight for functioning in bright and sunny condition and the battery power for functioning at night or in cloudy or foggy condition. Sensors used in the Wireless Sensor Network for traffic signal systems are mainly of two types: i) Intrusive type and ii) Non-Intrusive type.[10] i) Intrusive types of sensor are kept under the road and sense the traffic waiting at the signal. This type of sensor has the same working principle as that of a metal detector. ii) Non-Intrusive types of sensor is fitted on the road. The installation of this type of sensor is easy as no cutting of road is needed to be done. Non-intrusive sensor includes acoustic sensors or video image processors to detect the presence of vehicles waiting at the traffic intersection. Although Intrusive sensors are very effective still Non-intrusive sensors are preferred over Intrusive sensors as they are cost-effective, easy to install, immune to natural corrosion and degradation.

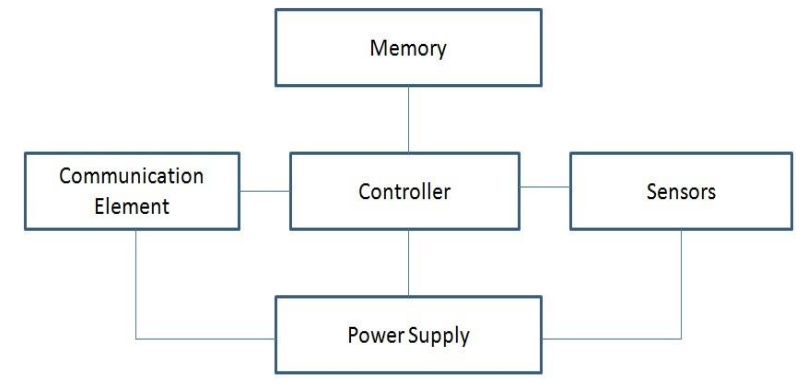

Figure 1.1 Typical Sensor Node Architecture.

Several accident cases on traffic light have been reported in past due to poor control of traffic signal at cross roads. In today's world speed is the ultimate word. Everyone is running a rat race and people definitely prefer to spend more time and utilize their energy in doing their respective professional and personal work rather than wasting both their valuable time and energy in commuting on road. This is the main reason behind accidents that people are in hurry and they do rash driving to reach their destinations and save time; and in the process of rash driving, they often make mistakes which result in fatal accidents especially at crossings where $\mathrm{n}$ intersections are meeting.[6] It is also seen that not all accidents are due to the fault of the driver of the vehicle making the accident, many a times it is due to the fault of other fellow people on the road.

Suppose a vehicle has to travel a hundred miles to reach its destination via some city and on its way it has to pass through numerous intersections of city. If the traffic system at those intersections is controlled by preset timers then the vehicle will waste precious time at most intersections. A traffic intersection is shown in figure 1.1.

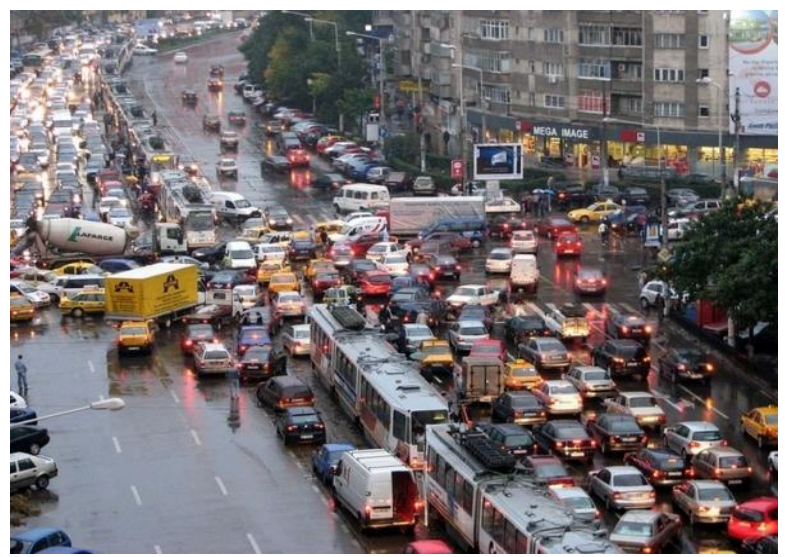

Fig 1.2 Heavily Traffic Loaded Intersection. 
Especially in case of long journey in order to save time there might arise a tendency in the driver to break the traffic intersection rule and drive even though the signal light is red. This can result in accident or unnecessary chaos. Previously a lot of work has been done in measuring traffic volume in a lane [4] and hence making the traffic signal adaptive at an intersection. A lot of work has been done to make traffic intersections adaptive but increased load of traffic looking for more refinement in intersections.

Research has shown that local administrative authorities has done study of traffic at local regional level to make smooth movement of traffic and accordingly these authorities have made traffic signals with static time limit to pass. More time is given for that intersection traffic where more traffic load is observed and this time increase or decrease in different time of day as per the traffic load to give more adaptive-ness to intersections. But this work is not enough to handle traffic adaptively; hence work on reducing waiting time on traffic signal is done.

This paper is divided into 4 sections. Second section focus on the existing approaches in field of intelligent traffic light system. Third section focus on the proposed method and fourth section is for further scope of research in this domain.

\section{EXISTING APPROACHES:}

D-WAN: This method states that vehicles have some active communication system through which a driver can communicate to the traffic intersection point regarding the lane in which he should drive to reach its destination. On other hand intersection point guides the vehicle driver to take appropriate turn in order to reach his destination. The whole idea proposed in this paper was to make vehicle intelligent so that they can communicate with the traffic system in advance before reaching to that intersection and get information about the path to reach destination. But this method does not take care for the load of traffic on a particular intersection and adaptation of traffic light as per the traffic load. Vehicles keep on waiting even if no vehicle has to pass from other's intersection side.[9]

Reducing Waiting Time: This paper is fully focused to make the traffic light fully adaptive using the wireless sensor network. According to the concept proposed in this paper, traffic light change their states from Red to Green or vice versa as per the load of traffic and static time limit has the least importance as load of traffic over rule the traffic signal automatically. Although to control the traffic an upper limit of green signal was set but wastage of green time was minimum. But, this paper does not focus upon the prioritization of vehicle on intersections because at some point of times it happens that emergency vehicles keep on waiting for their intersection side to get green and clear to pass. And it has been seen that some time due to this, rescue team gets delay to reach their destinations which results in more damage. Hence, this need bit refinement to make some changes which takes care for the emergency vehicles to get pass with priority. [2]

Vehicle Based Mobile Sensor Network for Traffic Monitoring: In this literature two types of algorithms were proposed: i) link-based algorithm and ii) vehicle based algorithm. In the link based algorithm it is assumed that if a particular link is given then, then a pair of sensors situated at the starting point and at the ending point of the link can best reflect the traffic status of that link. In contrary the vehicle based algorithm uses every available data pair to consider all the links travelled by them to calculate an average speed of the traffic. Thus, we can say that a sensor fitted in a vehicle can travel across more than one link which in turn can again be associated with more than one road. The result gathered can be accurate but having sensors in all the vehicles and also on all the roads is very costly especially when we are taking into consideration an economically poor country like India.[8]

WSITMN: WSITMN is a traffic monitoring system that uses Radio Frequency Identification (RFID) tags and Wireless Sensor Network (WSN).[7] Here every vehicle is given a RFID tag and when a vehicle with a RFID tag enters the monitoring zone, the RFID reader reads the information on the RFID tag and gathers information about the traffic flow. All the data gathered are then processed and sent to the base station. The base station then compiles the data, and transmits it to the monitoring center, where all the data gathered are analyzed and a decision is taken. Here the drawback is that the main monitoring center is a centralized system and if it fails then the whole system becomes useless. This process is also a bit time taking and it is also not possible to set RFID tags on all the passing vehicles.[3]

\section{PROPOSED METHOD}

From the literatures it can be concluded that full automation is required for making smart traffic control system which good take care for the emergency vehicles. In a country like India, where the economic condition is in development stage and the existing traffic signal infrastructure is non-automated and making the whole traffic signal system automated is not possible. So, the proposed idea takes this problem into account and uses the existing infrastructure to fulfill the criteria.

In this paper, the primary aim is to gather the information of moving emergency vehicles based on WSN to provide them a clear path till their destinations and traffic signals should switch automatically to give a clear way for these emergency vehicles. The adaptive traffic signal system that can do the following:

- Intelligent traffic signal system based on the volume of traffic on each side of the signal.

- Optimize the following:

- Minimize the average waiting time.

- Maximize the average number of vehicles passing through the intersection.

- Minimizing the number of accidents that occur due to red light violation.

- Prioritization of vehicles serving emergency purpose like ambulance, fire brigade, rescue vehicles etc. and VIP vehicles. 
In the proposed method, traffic intersection will be able to communicate with the next neighboring intersection signals in order to send and receive message about any emergency vehicle movement which is hereafter will be refer as II (Intersection to Intersection) communication. Sensors are also installed on the road between two intersections of a road which will help to intersection about the movement of emergency vehicle. To illustrate this, consider figure 3.1.

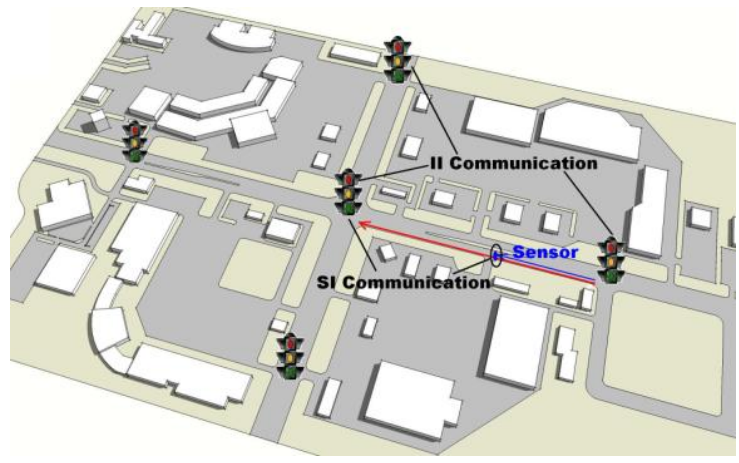

Figure 3.1 SI and II communication system.

But this concepts leads to another problem and that is what if all four sides of intersection or more than one side of intersection is loaded with emergency vehicle and approaching towards the common traffic intersection. In such case, there will an ambiguous situation for traffic signal to decide that, to which side it should give green signal or clear way.

To solve this kind of situation, whenever any emergency vehicle passes from road then the road side sensors will detect the vehicle based on sound system and this sensor will trigger an event to next traffic intersection point informing that a emergency vehicle has to pass and give a clear way with immediate effect which is hereafter refer as SI (Sensor To Intersection) communication. As soon as intersection will receive any signal from road side signals then it will give Red signal to remaining intersection sides and give green to that side from where sensor has triggered that event.

Road side sensors have different sound detection capability and they can distinguish the sound emitted from the different emergency purpose vehicles. These sensors are customized to trigger event when the sound emitted by the emergency vehicles exceed a certain intensity level. This property helps to distinguish between ordinary vehicles and emergency purpose vehicles and then the sensor triggers the event.

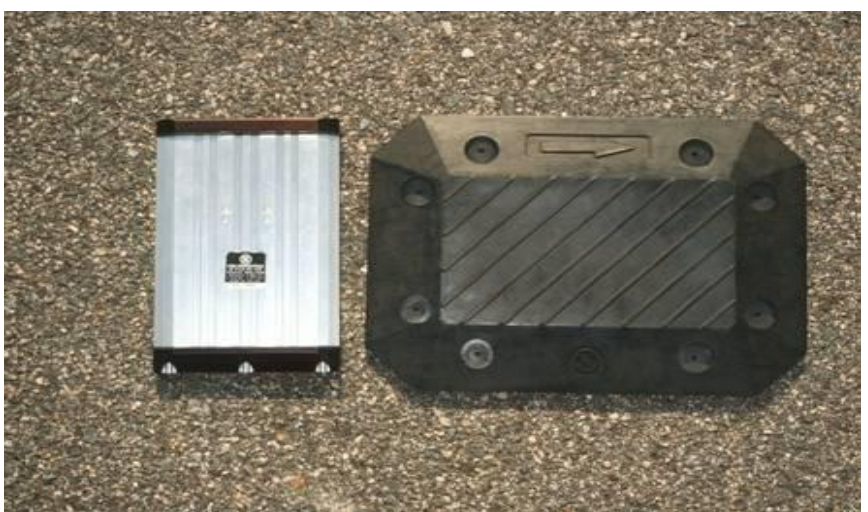

Figure 3.2 (a) A sensor.

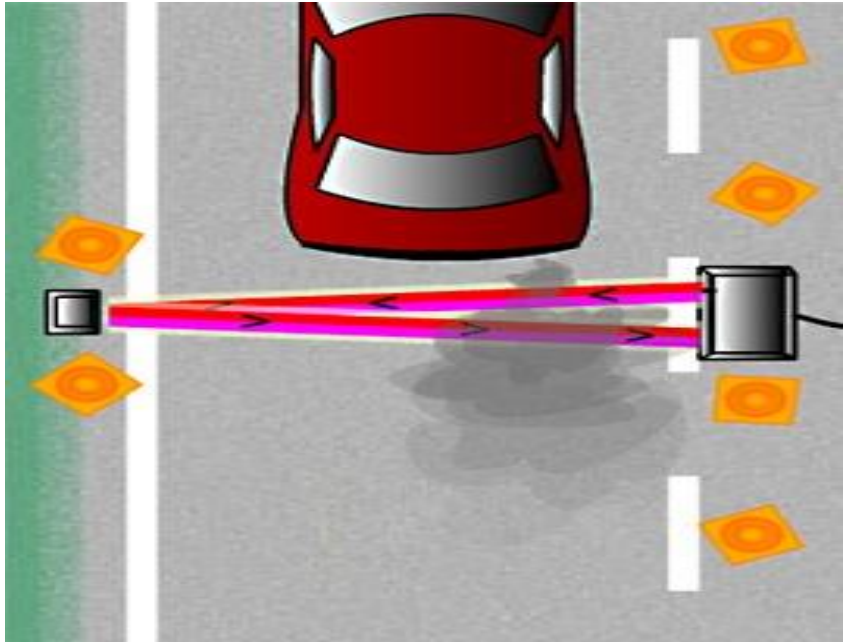

Figure 3.2 (b) Line of sight sensor and vehicle is about to cross sensor line.

So, if two sides or more than two side of common intersection point is loaded with emergency vehicle then in such case, intersection will serve on first come first serve basis and will give clear way to that side from where it will receive first trigger event. As, intersection will maintain the information in buffer about other trigger events on same or nearby moment, and as soon as one way will be clear it will automatically allow to second side traffic to pass on. Once the vehicle will pass from the intersection, then intersection will clear the buffer. In this way, ambiguity of passing vehicle from more than one side can be resolved.

As soon as that vehicle will pass from the intersection then intersection will inform to next neighboring intersections about movement of emergency vehicle using II communication. According to II communication, as soon as any vehicle will pass from intersection then immediately that intersection will communicate to next neighboring intersection about the movement of emergency vehicle and if corresponding road side signal will trigger an event then they will give a green way to that side.

Position of sensor on road will depend on the gap between two consecutive intersections on road. For example, if two intersections are $1000 \mathrm{mtrs}$ apart then sensors can be placed after 400mtr of intersection so that when any emergency vehicle passes from there then that sensor will detect the vehicle and within microsecond it will indirectly enable the green on that side. Time take by vehicle to travel $600 \mathrm{mtrs}$ from sensor position to intersection point will be around 30 seconds on average and 30 seconds will be enough to clear the buffered traffic in average situation. This analogy of sensor position can be better understood by figure 3.1 where red line is taken up of $1000 \mathrm{mtrs}$ and blue line is of $400 \mathrm{mtrs}$. On 400 mtrs there is a blue circle which is indicating a road side sensor that is responsible to communicate with the intersection point. We have taken the example of up road, and in the similar manner sensors can be install a down side road. More clear view of road side sensor is shown in figure 3.2 (b).

If in any case gap between two intersection is less than or equal to $600 \mathrm{mtr}$ then in such situations, II communication will come into picture and no road side sensors will be needed. Road side sensors can also be avoid on those roads which are equipped with emergency purpose vehicle base stations like Hospital, Fire Brigade Station, Police Station etc. because if road side sensors are installed on these roads then this can 
lead to unnecessary green light even when not in need. So, to customize this situation, road side sensors can be placed on the exit gate of these base stations instead of road side; and whenever any vehicle exits from their base station then they can get a clear way on immediate next traffic light. If any SI communication happens and that side is already on green mode then that green light will remain active until that vehicle does not pass.

\section{CONCLUSION}

This paper touched on key point to give a clear way to emergency purpose vehicles on road so that they can reach to their destination in least time by not stopping at the traffic intersections. Traffic intersections will be smart enough to take care for flow of traffic if there is any emergency purpose vehicle need to pass on and in normal condition, traffic intersection will work normally as discussed in earlier paper. But a practical implementation of this paper need to grass root level and then optimization can be done accordingly.

\section{REFERENCES}

[1] Wenjie Chen, Lifeng Chen, Zhanglong Chen, and Shiliang Tu.Wits. "A wireless sensor network for intelligent transportation system." in International MultiSymposiums of Computer and Computational Sciences Conference (IMSCCS'06), pages 635-641, April 2006.

[2] Amnesh Goel, Sukanya Ray, Nidhi Chandra "Minimization of Waiting Time in Traffic Signals on Indian roads based on Wireless Sensor Network" JOURNAL OF COMPUTING, VOLUME 3, ISSUE 12, DECEMBER 2011, ISSN 2151-9617

[3] Sharma, A., Chaki, R., Bhattacharya, U. "Applications of Wireless Sensor Network in Intelligent Traffic System: A Review" Electronics Computer Technology (ICECT), 2011 3rd International Conference on Issue Date: 8-10 April 2011 On page(s): 53 - 57 Print ISBN: 978-1-42448678-6
[4] Abishek C, Mukul Kumar and Kumar Padmanabh, "City Traffic Congestion Control in Indian Scenario using Wireless Sensors Network", Fifth IEEE Conference on Wireless Communication and Sensor Networks (WCSN), 2009, pp. 1-6.

[5] Malik Tubaishat, Qi Qi, Yi Shang, Hongchi Shi "Wireless Sensor-Based Traffic Light Control" IEEE CCNC 2008 proceedings 1-4244-1457-1/08

[6] Qingfeng Huang and Ying Zhang. "Dynamic balancing of push and pull in a distributed traffic information system." In IEEE Consumer Communications and Networking Conference (CCNC 2007), 2007.

[7] Jianhou Gan, Lingyun Yuan, Zhongqi Sheng and Tianwei $\mathrm{Xu}$, "Construction and Implementation of an Integrated WSID Traffic Monitoring Network System", Proc. 21st annual international conference on Chinese control and decision conference, 2009, pp. 4726-4731.

[8] Xu Li, Wei Shu, Minglu Li, Hong-Yu Huang, Pei-En Luo, Min-You Wu, "Performance Evaluation of Vehicle-Based Mobile Sensor Networks for Traffic Monitoring" IEEE transactions on vehicular technology, May 2009, vol. 58, no. 4, pp. 1647-1653.

[9] Ben-Jye Chang, Bo-Jhang Huang and Ying-Hsin Liang, "Wireless Sensor Network-based Adaptive Vehicle Navigation in Multihop-Relay WiMAX Networks", Proc. 22nd International Conference on Advanced Information Networking and Applications (AINA), 2008, pp. 56-63.

[10] Sensor node information available via www at en.wikipedia.org/wiki/Traffic_light_control_and_coordin ation. 